\title{
Facteurs personnels, scolaires et familiaux différenciant les garçons en problèmes de comportement du secondaire qui ont décroché ou non de l'école
}

\author{
Laurier Fortin, Diane Marcotte, Égide Royer et Pierre Potvin
}

Numéro hors-série, 2018

Numéro spécial $25^{\mathrm{e}}$ anniveraire

URI : https://id.erudit.org/iderudit/1059212ar

DOI : https://doi.org/10.7202/1059212ar

Aller au sommaire du numéro

Éditeur(s)

Faculté d'éducation, Université de Sherbrooke

ISSN

1911-8805 (numérique)

Découvrir la revue

Citer cet article

Fortin, L., Marcotte, D., Royer, É. \& Potvin, P. (2018). Facteurs personnels, scolaires et familiaux différenciant les garçons en problèmes de comportement du secondaire qui ont décroché ou non de l'école. Nouveaux cahiers de la recherche en éducation, 85-97. https://doi.org/10.7202/1059212ar
Résumé de l'article

L'objectif de cette étude est d'identifier les facteurs personnels, familiaux et scolaires différenciant les élèves en problèmes de comportement décrocheurs et non décrocheurs. Les participants sont 360 élèves du secondaire provenant d'une étude longitudinale sur les difficultés d'adaptation sociale et scolaire. Ils ont été évalués sur un très grand nombre de variables personnelles, familiales et scolaires à partir de leur première secondaire. Au cours des cinq années du secondaire, 150 élèves ont présenté des problèmes de comportement et 45 d'entre eux ont décroché à l'âge de 20 ans. Les résultats montrent que les élèves en problèmes du comportement décrocheurs se différencient de ceux non décrocheurs sur plusieurs variables personnelles (coopération), familiales (encadrement) et scolaires (réussite scolaire, attitudes des enseignants). 


\section{Facteurs personnels, scolaires et familiaux différenciant les garçons en problèmes de comportement du secondaire qui ont décroché ou non de l'école}

\section{Laurier Fortin}

Université de Sherbrooke

\section{Diane Marcotte}

Université du Québec à Montréal

\section{Égide Royer}

Université Laval

\section{Pierre Potvin}

Université du Québec à Trois-Rivières

\section{Résumé}

L'objectif de cette étude est d'identifier les facteurs personnels, familiaux et scolaires différenciant les élèves en problèmes de comportement décrocheurs et non décrocheurs. Les participants sont 360 élèves du secondaire provenant d'une étude longitudinale sur les difficultés d'adaptation sociale et scolaire. Ils ont été évalués sur un très grand nombre de variables personnelles, familiales et scolaires à partir de leur première secondaire. Au cours des cinq années du secondaire, 150 élèves ont présenté des problèmes de comportement et 45 d'entre eux ont décroché à l'âge de 20 ans. Les résultats montrent que les élèves en problèmes du comportement décrocheurs se différencient de ceux non décrocheurs sur plusieurs variables personnelles (coopération), familiales (encadrement) et scolaires (réussite scolaire, attitudes des enseignants).

Originalement paru dans:

Nouveaux cahiers de la recherche en éducation, vol. 8, no 2, 2005, p. 79 à 88 


\section{Abstract}

The goal of this study was to identify personal, family, and academic factors that differentiate pupils with behavioural problems who drop out of school from those who do not. Participants in the study were 360 secondary-school pupils taking part in a longitudinal study of social and academic adjustment difficulties. They were assessed, as of their first year of secondary school, for a very large number of personal, family, and academic variables. Over the five years of secondary school, 150 pupils presented with behavioural problems and 45 of these dropped out at age 20. Our findings show that pupils with behavioural problems who drop out can be distinguished from those that don't by several variables of a personal kind (cooperation), a family kind (supervision) and an academic kind (academic success, teacher attitudes). 


\section{Problématique}

Le décrochage scolaire au secondaire est un problème social majeur au Québec. Le ministère de l'Éducation du Québec (MEQ) (Gouvernement du Québec, 2004) estime que 34,2 \% des jeunes de 20 ans et moins n'ont pas obtenu de diplôme d'études secondaires en 2002-2003. En analysant les données selon le genre, 41,9 \% des garçons n'ont pas obtenu leur diplôme. Le MEQ (Gouvernement du Québec, 2000) définit le décrocheur comme celui qui quitte l'école sans avoir obtenu de diplôme d'études secondaires et qui n'est pas inscrit dans aucune école secondaire. Les conséquences pour les jeunes décrocheurs sont très lourdes. Au plan personnel, plusieurs jeunes présentent des inadaptations sociales telles que des problèmes du comportement et de la délinquance (Battin-Pearson, Newcomb, Abbott, Hill, Catalano et Hawkins, 2000; Fortin et Picard, 1999). Au plan social et économique, ils n'ont pas de formation technique et ils éprouvent de grandes difficultés à s'insérer dans le monde du travail. En outre, ils présentent un taux de chômage très élevé et ils bénéficient davantage de l'aide sociale et de l'assurance emploi que les jeunes diplômés (Garnier, Stein et Jacobs, 1997).

Plusieurs chercheurs estiment que les problèmes du comportement des élèves sont un puissant prédicteur du décrochage scolaire (Battin-Pearson et al., 2000; Jimerson, Egeland, Sroufe et Carlson, 2000; French et Conrad, 2001). Fortin et Picard (1999) montrent que les élèves à risque de décrochage scolaire qui demeurent dans le système scolaire manifestent moins de délinquance sociale et moins de problèmes du comportement que ceux qui ont abandonné leurs études. Fortin, Royer, Potvin, Marcotte et Yergeau (2004) rapportent un lien entre les délits, les agressions, les lacunes d'habiletés sociales et le risque de décrochage scolaire. Walker, Colvin et Ramsey (1995) observent qu'un élève qui a dix notes disciplinaires ou plus documentées au bureau du directeur d'école pendant une année scolaire est fortement à risque d'échec scolaire, de délinquance, de consommation d'alcool et de drogues, et il démontre une forte possibilité d'éventuels problèmes sociaux. Tobin et Sugai (1999) montrent que le nombre de notes disciplinaires agit comme élément précurseur de l'obtention ou non du diplôme. Enfin, le décrochage scolaire est un long processus, cumulatif et hiérarchisé, qui s'explique à partir de facteurs personnels mais aussi familiaux et scolaires.

Battin-Pearson et al. (2000) rapportent que le peu d'attentes des parents envers la réussite scolaire est une variable fortement associée au décrochage de leur enfant. Jimerson et al. (2000) observent que la qualité de l'environnement familial et la qualité des soins dispensés à l'enfant sont de puissants prédicteurs de la réussite scolaire ou du décrochage. Le manque de supervision et les pratiques éducatives inadéquates sont aussi rapportées (McNeal, 1999). À cet égard, Potvin, Deslandes, Beaulieu, Marcotte, Fortin, Royer et Leclerc (1999) montrent que les pratiques éducatives des parents telles que 
le peu de soutien affectif, le peu d'engagement dans les activités scolaires et le peu d'encadrement sont fortement associées au risque de décrochage scolaire.

Nombre d'études montrent que les élèves évoquent souvent les expériences négatives vécues à l'école pour expliquer le décrochage. Les élèves qui ont choisi les raisons scolaires comme raisons principales de décrochage décrivent un cheminement scolaire particulièrement difficile, soit en lien avec le rendement scolaire faible, les conflits avec les enseignants, l'ennui et le manque de motivation (Lessard, Fortin, Joly, Royer, Potvin et Marcotte, 2006). Les élèves en problèmes du comportement seraient plus affectés par un environnement éducatif lacunaire que les élèves qui possèdent de bonnes compétences intellectuelles et sociales (Janosz, Georges et Parent, 1998). Smyth et Hattam (2002) estiment que certaines écoles adoptent une voix hostile qui se traduit par l'expulsion de l'élève qui est dérangeant dans l'école. Lee et Burkam (2003) identifient aussi que des écoles peuvent contribuer au décrochage scolaire en expulsant les élèves indésirables.

En résumé, de nombreuses études rapportent que les élèves en problèmes du comportement à l'école sont fortement associés au décrochage scolaire. Par contre, nous savons que ce n'est pas tous les élèves en problèmes de comportement qui décrochent, car un certain nombre réussissent à obtenir leur diplôme d'études secondaires. Quels sont les facteurs personnels, familiaux et ceux associés à l'école qui différencient les élèves présentant des problèmes du comportement qui ont décroché de ceux qui n'ont pas décroché? Nous estimons qu'une meilleure connaissance de ces facteurs permettra de mieux dépister les élèves à risque de décrochage scolaire et de mettre en place des programmes de prévention du décrochage mieux adaptés et plus prometteurs ${ }^{1}$. L'objectif est de comparer les caractéristiques personnelles, familiales et scolaires entre les élèves qui présentent des problèmes de comportement et qui ont décroché, ceux qui n'ont pas décroché et les élèves d'un groupe témoin lorsqu'ils étaient en première secondaire.

\section{Méthode}

\subsection{Participants}

L'échantillon provient de l'étude longitudinale sur les difficultés d'adaptation scolaire et sociale de Fortin, Marcotte, Royer et Potvin (1996-2005). En 1996, l'échantillon comptait 808 élèves provenant de la ville de Québec (151 élèves), de Trois-Rivières (346 élèves) et de Sherbrooke (217 élèves). Les participants étaient âgés entre 12 et 13 ans et il y avait $53,9 \%$ de garçons et $46,1 \%$ de filles. À l'aide des indicateurs socioéconomiques du ministère de l'Éducation du Québec (Gouvernement du Québec, 2002), les cohortes de Sherbrooke et de Québec sont situées dans un quartier socioéconomique plutôt défavorisé

$\overline{1}$ Cette étude a été réalisée grâce aux fonds du Conseil de recherche en sciences humaines du Canada (CRSH) et du Conseil québécois pour la recherche sociale (CQRS). 
alors que celle de Trois-Rivières est dans un quartier de classe sociale moyenne. En 1996, 360 garçons étaient âgés de 12-13 ans en première secondaire. Pour chacun des cinq temps de mesure, le critère de problèmes du comportement a été attribué lorsque le score d'un jeune à l'échelle de problèmes de comportement extériorisés du "Questionnaire d'habiletés sociales " (Gresham et Elliott, 1990) se situait au-delà d'un écart-type du score moyen du groupe de garçons à chaque temps de mesure donné. À partir de ce critère, 150 garçons ont présenté des problèmes du comportement sur une période d'au moins un an au cours des cinq années du secondaire, alors que 210 n'en ont présenté aucun. Parmi les garçons en problèmes du comportement, 105 n'avaient pas décroché et 45 avaient décroché de l'école à l'âge de 20 ans.

\subsection{Mesures}

"Délinquance auto-révélée" - Cette mesure (LeBlanc, 1994) comprend 29 questions répondues par le jeune et elle évalue les délits et diverses activités délinquantes (vandalisme, vols mineurs, vols graves, agressions physiques, consommation de drogues, consommation d'alcool et autres comportements). L'alpha standardisé est de 0,82 . Ce questionnaire montre une très bonne validité discriminante, car les adolescents délinquants se distinguent des adolescents conventionnels sur la très grande majorité des échelles et des sous-échelles.

"Inventaire de dépression de Beck" - Traduit en français par Bourque et Beaudette (1982), cette mesure (Beck, 1978) auto-rappportée comprend 21 items permettant d'évaluer l'intensité des symptômes affectifs, comportementaux, cognitifs et somatiques de la dépression. Les qualités psychométriques de l'inventaire ont été confirmées auprès d'adolescents québécois, les coefficients de consistance interne variant entre 0,86 et 0,88.

"Questionnaire sur les habiletés sociales" - Version française du Social Skills Rating System (Gresham et Elliott, 1990), ce questionnaire répondu par l'enseignant fournit une évaluation multivariée des comportements sociaux des jeunes à l'école telles que les habiletés sociales (coopération, contrôle de soi et affirmation de soi) et les problèmes du comportement extériorisés. Des analyses factorielles en composantes principales avec rotation varimax auprès de 810 élèves du secondaire indiquent que la version québécoise possède la même structure factorielle que la version américaine (Fortin, Royer, Marcotte, Potvin et Joly, 2001). Les coefficients de consistance interne varient entre 0,79 et 0,92.

"Les stratégies d'adaptation" - Ce questionnaire (Lazarus et Folkman, 1984) a été traduit et adapté par Bouchard, Sabourin, Lussier, Richer et Wright (1995). II mesure trois types de stratégies d'adaptation, soit la recherche de soutien social, la réévaluation positive/ résolution de problèmes et la distanciation/évitement. La version abrégée répondue par 
le jeune comprend 21 items. Des coefficients de consistance interne variant entre 0,61 et 0,81 ont été obtenus avec des échantillons d'adolescents québécois.

"Analyse des dossiers scolaires" - Le dossier permet de colliger les résultats scolaires en français, en mathématiques et l'absentéisme scolaire.

"Échelle de l'environnement de la classe» - Traduction française du Classroom Environment Scale (Moos et Trickett, 1987), cet instrument répondu par l'élève permet d'évaluer le climat social de la classe. La version abrégée comprend 45 énoncés de 9 échelles (engagement, lien d'appartenance aux pairs, soutien de l'enseignant, orientation au travail, compétition, ordre et organisation, clarté des règlements, contrôle de l'enseignant, innovation pédagogique). La cohérence interne est adéquate (alpha de Cronbach variant entre 0,52 et 0,75 ) ainsi que la validité de convergence avec d'autres instruments ( $r$ de 0,16 à 0,40).

"Échelle d'attitudes du professeur envers le jeune" - Cet inventaire (Potvin et Rousseau, 1991) répondu par l'enseignant évalue les attitudes des enseignants. Il est composé de 18 adjectifs bipolaires dont chaque paire peut prendre une valeur de -3 à +3 avec un total minimum de -54 et un maximum de +54 . Il permet d'évaluer les attitudes positives ou négatives de l'enseignant envers l'élève. La fidélité de l'instrument est adéquate (alpha de 0,50 à 0,86 ) et le degré de corrélation des items est de 0,51 à 0,94.

"Style parental» -Version française de Parental Style (Steinberg, Lamborn, Dornbusch et Darling, 1992), ce questionnaire répondu par l'élève comprend trois échelles: I'engagement parental (12 items), l'encadrement parental (6 items) et l'encouragement à l'autonomie (6 items). Des analyses factorielles en composantes principales avec rotation oblique auprès de 145 élèves du secondaire indiquent que la version québécoise se compare à la version américaine. Les indices de consistance interne sont tout à fait satisfaisants (alpha de 0,71 à 0,87) (Deslandes, Bertrand, Royer et Turcotte, 1995).

"Participation parentale au suivi scolaire" - Ce questionnaire (Epstein, Connors et Salinas, 1993) a été traduit et validé au Québec par Deslandes et al. (1995). Il est composé de 20 énoncés répondus par l'élève et évaluant la participation des parents dans 20 activités à la maison ou à l'école. Ces énoncés sont répartis en cinq sous-échelles : le soutien affectif des parents (alpha de 0,78 ), la communication avec les enseignants (alpha de 0,68 ), les interactions entre parents et adolescents (alpha de 0,73 ), la communication parents-école (alpha de 0,56), et la communication parents-adolescents (alpha de 0,64).

\subsection{Procédure}

Tous les participants ont été joints par l'entremise de l'enseignant. Ce dernier a été informé de l'étude par le directeur d'école puis rencontré par un des expérimentateurs afin 
de lui expliquer les objectifs de l'étude et pour solliciter sa participation. Puis, les parents ou tuteurs légaux ont été approchés afin d'obtenir leur consentement écrit et leur garantir la confidentialité des informations recueillies. Le pourcentage d'élèves qui ont accepté de participer était de 78,1\%. Les questionnaires ont été administrés en groupe classe à tous les jeunes par des assistants de recherche au cours des mois d'octobre et de novembre et au cours des mois de mars et d'avril de chaque temps de mesure et en même temps dans les trois sites à chaque année pendant les cinq années du secondaire. Chaque temps de mesure représente une année scolaire et comprend les deux évaluations. L'enseignant responsable du groupe a complété les questionnaires d'habiletés sociales et l'échelle d'attitudes du professeur pour chacun des jeunes de son groupe au cours du mois d'avril. Le décrochage des élèves est identifié à partir du code permanent de l'élève et de la banque d'information du ministère de l'Éducation du Québec huit ans après le début du secondaire du jeune alors qu'il avait 20 ans.

\section{Résultats}

L'objectif est de comparer les caractéristiques personnelles, familiales et scolaires entre les élèves qui présentent des problèmes du comportement qui ont décroché, ceux qui n'ont pas décroché et les élèves d'un groupe témoin lorsqu'ils étaient en première secondaire. Les résultats au test du chi-carré montrent une valeur de $33,20(p=0,00)$, ce qui signifie que le groupe en problèmes du comportement décrocheurs se différencie significativement du groupe d'élèves témoins. Il est intéressant de noter que ce ne sont pas tous les élèves en problèmes du comportement qui décrochent, car il y avait $70 \%$ de ces derniers qui n'ont pas décroché. Parmi ceux qui ont décroché, $33 \%$ des élèves présentaient des problèmes de comportement stables, c'est-à-dire à plus d'un temps de mesure. Aussi, $24 \%$ des élèves avaient résolu leurs problèmes de comportement au cours du secondaire, $29 \%$ des élèves avaient commencé leurs problèmes du comportement à partir de la troisième secondaire et $13 \%$ ont présenté des problèmes de comportement sur une base instable pendant les cinq années du secondaire. Enfin, 90,2 \% des élèves témoins n'avaient pas décroché. Le tableau 1 présente les moyennes obtenues pour chacune des caractéristiques des trois sous-groupes d'élèves. Étant donné le très grand nombre de variables à l'étude, nous avons appliqué la correction Bonferonni pour éviter le risque d'erreur de type 1 qui est d'identifier des différences entre les groupes d'élèves en l'absence de telles différences. Par conséquent, le seuil de signification des analyses a été fixé à $\mathrm{p}<0,002$. Mais, un effet possible de cette correction est de commettre l'erreur de type 2 qui est de ne pas identifier de différence entre les groupes alors qu'il y en a une. 
Tableau 1

Comparaison entre les différents sous-groupes d'élèves: analyses de la variance sur les variables personnelles, familiales et scolaires (Anova)

\begin{tabular}{|c|c|c|c|c|c|}
\hline \multirow[t]{2}{*}{ Variables dépendantes } & \multicolumn{3}{|c|}{ Moyennes (é.t.) } & \multirow[t]{2}{*}{$\mathbf{F}$} & \multirow{2}{*}{$\begin{array}{c}\text { Tests } \\
\text { post hoc } \\
\text { (Gabriel) }\end{array}$} \\
\hline & $\begin{array}{l}\mathrm{PC} \text { décl| } \\
\mathrm{N}=45\end{array}$ & \begin{tabular}{|c|} 
PC non décr. \\
$\mathrm{N}=105$
\end{tabular} & $\begin{array}{l}\text { Témoins } \\
\mathrm{N}=210\end{array}$ & & \\
\hline \multicolumn{6}{|l|}{ Variables personnelles } \\
\hline Q.I. & $41(25,63)$ & $65(24)$ & $75(23)$ & 28,60 & $1<2$ et 3 \\
\hline Délitsa & $6,38(4,27)$ & $5,72(4,21)$ & $3,80(3,27)$ & $10,02^{*}$ & $1>3$ et $2>3$ \\
\hline Agressions physiques & $2,07(1,39)$ & $1,72(1,38)$ & $1,19(1,34)$ & $7,90^{*}$ & $1>3$ et $2>3$ \\
\hline Dépression & $7,82(8,97)$ & $8,00(9,18)$ & $6,45(5,86)$ & 1,125 & \\
\hline Coopération $^{a}$ & $12,09(4,79)$ & $15,44(3,77)$ & $16,97(3,59)$ & $24,77^{*}$ & $1<2<3$ \\
\hline Contrôle de soi & $12,36(3,61)$ & $13,05(3,84)$ & $14,52(4,02)$ & $6,40^{*}$ & $1<3$ et $2<3$ \\
\hline Affirmation de soi & $9,79(3,56)$ & $10,45(4,56)$ & $8,71(4,35)$ & 4,37 & \\
\hline Distanciation/évitement & $1,46(0,59)$ & $1,46(0,55)$ & $1,31(0,61)$ & 2,26 & \\
\hline Réévaluation positive/résolution problèmes & $1,72(0,65)$ & $1,68(0,52)$ & $1,60(0,60)$ & 0,86 & \\
\hline \multicolumn{6}{|l|}{ Variables scolaires } \\
\hline Notes en français & $64,56(8,70)$ & $71,20(8,47)$ & $74,14(8,81)$ & $19,43^{*}$ & $1<2<3$ \\
\hline Notes en mathématiques & $65,36(12,47)$ & $76,73(11,63)$ & $81,02(10,95)$ & $28,89^{*}$ & $1<2<3$ \\
\hline Engagement (classe) & $1,93(1,30)$ & $1,91(1,32)$ & $2,22(1,22)$ & 1,77 & \\
\hline Lien d'appartenance aux pairs & $2,79(1,07)$ & $2,93(1,09)$ & $3,28(0,92)$ & 4,77 & \\
\hline Soutien de l'enseignant & $2,16(1,25)$ & $2,22(1,41)$ & $2,47(1,32)$ & 1,25 & \\
\hline Orientation travail & $2,61(1,08)$ & $2,74(1,02)$ & $2,97(0,96)$ & 2,52 & \\
\hline Compétition & $1,36(0,84)$ & $1,46(0,86)$ & $1,34(0,81)$ & 0,63 & \\
\hline Ordre et organisation & $1,49(1,20)$ & $1,78(1,31)$ & $2,03(1,38)$ & 2,80 & \\
\hline Clarté des règlements & $2,79(1,04)$ & $3,02(1,02)$ & $3,19(0,95)$ & 2,64 & \\
\hline Contrôle de l'enseignant & $2,52(1,30)$ & $2,31(1,24)$ & $1,89(1,26)$ & 5,14 & \\
\hline Innovation & $2,33(1,17)$ & $2,00(1,12)$ & $2,26(1,14)$ & 1,98 & \\
\hline Attitudes de l'enseignant ${ }^{a}$ & $11,31(19,80)$ & $24,64(18,91)$ & $33,39(16,13)$ & $24,54^{*}$ & $1<2<3$ \\
\hline 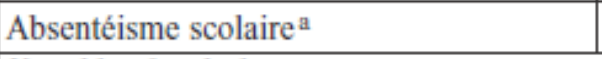 & $25,84(31,07)$ & $12,27(12,06)$ & $11,51(18,14)$ & $10,06^{*}$ & $1>2$ et $1>3$ \\
\hline \multicolumn{6}{|l|}{ Variables familiales } \\
\hline Encadrement parental & $2,01(, 44)$ & $2,31(, 46)$ & $2,23(, 49)$ & $6,54^{*}$ & $1<2$ et $1<3$ \\
\hline Encouragement à l'autonomie & $2,35(0,64)$ & $2,26(, 57)$ & $2,45(50)$ & 2,92 & \\
\hline Interaction scolaire parents-jeune & $3,02(59)$ & $3,31(56)$ & $3,15(61)$ & 4,46 & \\
\hline Communication parents-école & $2,13(71)$ & $2,25(68)$ & $2,17(65)$ & 0,57 & \\
\hline Communication parents-adol. & $2,24(0,67)$ & $2,31(63)$ & $2,30(68)$ & 0,20 & \\
\hline Soutien affectif & $2,79(58)$ & $2,98(62)$ & $2,94(66)$ & 1,32 & \\
\hline Communication avec les enseignants & $2,20(79)$ & $2,16(64)$ & $2,04(71)$ & 1,17 & \\
\hline
\end{tabular}

Légende: Correction Bonferroni: *p<0,002; COMPLÉTER signification du : a

Les analyses de la variance effectuées pour les variables personnelles, scolaires et familiales montrent des différences significatives selon les groupes de garçons pour neufs variables: les délits $(F=10,02 ; p=0,00)$, les agressions physiques $(F=7,90 ; p=0,00)$, la coopération ( $F=24,78 ; p=0,00)$, le contrôle de soi $(F=6,40 ; p=0,00)$, les notes en français $(F=19,43 ; p=0,00)$, les notes en mathématiques $(F=28,89 ; p=0,00)$, les attitudes de l'enseignant envers l'élève $(F=24,54 ; p=0,00)$, le taux d'absentéisme $(F=10,06 ; p=0,00)$ et l'encadrement parental $(F=6,5 ; p=0,00)$. Les analyses post-hoc montrent que les 
trois groupes se distinguent entre eux sur certaines variables personnelles, scolaires et familiales.

Au plan personnel, les jeunes en problèmes du comportement (PC) décrocheurs (décr.) et non décrocheurs (non décr.) rapportent en moyenne plus de délits ( $P C$ décr. $=6,38$; $P C$ non décr. $=5,72$; témoin $=3,80$ ) et ils montrent plus de comportements agressifs ( $\mathrm{PC}$ décr.=2,07; $P C$ non décr. $=1,72$; témoin $=1,19$ ) que les élèves sans problème du comportement. Sur ces dernières variables, les deux groupes en problèmes de comportement sont comparables. Aussi, le groupe témoin se distingue des deux groupes d'élèves en problèmes de comportement par de meilleures habiletés au plan du contrôle de soi ( $\mathrm{PC}$ décr.=12,36; PC non décr. $=13,05$; témoin $=14,52$ ). Les élèves du groupe en problèmes du comportement décrocheurs et en problèmes du comportement non décrocheurs se différencient entre eux et ils montrent moins de coopération que ceux du groupe témoin $(\mathrm{PC}$ décr.=12,09; PC non décr.=15,44; témoin=16,97).

Au plan scolaire, les élèves en problèmes du comportement décrocheurs présentent un taux d'absentéisme significativement plus élevé que les garçons en problèmes du comportement non décrocheurs ( $\mathrm{PC}$ décr.=25,81; PC non décr. $=11,51$ ) alors que les jeunes en problèmes du comportement non décrocheurs ne se différencient pas du groupe témoin sur ce plan ( $\mathrm{PC}$ non décr.=12,27; témoin=11,51). Les enseignants montrent plus d'attitudes négatives envers les groupes d'élèves en problèmes du comportement. Les enseignants expriment plus d'attitudes négatives envers le groupe en problèmes du comportement décrocheurs et un peu moins envers le groupe en problèmes du comportement non décrocheurs et ils expriment des attitudes positives envers ceux du groupe témoins (PC décr.=11,31; PC non décr.=24,64; témoin=33,39). Enfin, les trois groupes se différencient aussi au plan des résultats scolaires. Le groupe en problèmes du comportement décrocheurs présente les résultats les plus faibles en français (PC décr. $=64,56$; PC non décr. $=71,20$; témoin $=74,14)$ et en mathématiques (PC décr. $=65,36$; $P C$ non décr. $=76,73$; témoin $=81,02$ ) suivi du groupe en problèmes du comportement non décrocheurs. Les élèves du groupe témoin obtiennent les meilleurs résultats scolaires. Pour toutes les variables où il y a des différences significatives, le groupe en problèmes du comportement décrocheurs présente toujours un profil plus faible que le groupe PC non décrocheurs et ce dernier présente un profil plus bas que le groupe témoin.

$\mathrm{Au}$ plan familial, les résultats montrent une autre différence significative entre les élèves en problèmes de comportement décrocheurs et non décrocheurs sur la variable encadrement parental. En effet, les résultats indiquent un moins bon encadrement parental chez les élèves en problèmes du comportement décrocheurs par rapport à ceux du groupe en problèmes du comportement non décrocheurs et témoin (PC décr.=2,01; PC non décr.=2,31; témoin=2,23). Sur cette variable, le groupe témoin perçoit 
plus d'encadrement parental que le groupe d'élèves en problèmes du comportement décrocheurs.

\section{Discussion}

Dès le début du secondaire, les élèves en problèmes du comportement vivent plusieurs difficultés tant au plan personnel qu'aux plans scolaire et familial. Ces élèves sont ainsi exposés à un cumul de facteurs de risque qui augmentent considérablement leur niveau de difficultés d'adaptation scolaire et sociale et, par conséquent, la probabilité qu'ils décrochent au cours de leurs études secondaires. Les écrits scientifiques ainsi que les résultats de notre étude montrent qu'il y a un lien très fort entre les problèmes de comportement à l'école et le décrochage. Les problèmes de comportement qui se caractérisent par une plus grande stabilité sont généralement plus sévères et on pourrait croire que ce sous-groupe augmente significativement la proportion moyenne des décrocheurs. Les résultats de notre étude montrent que ce n'est pas le cas, car seulement le tiers des élèves composant ce sous-groupe ont décroché. Par conséquent, les décrocheurs proviennent aussi de sous-groupes qui peuvent se caractériser par de l'instabilité ou par de la discontinuité des problèmes du comportement au cours de leur secondaire.

Comparés aux élèves en problèmes du comportement non décrocheurs, ceux qui décrochent présentent de plus grandes difficultés en classe. Ces difficultés se traduisent par peu de coopération, par de faibles résultats scolaires, par un plus grand taux d'absentéisme et par des attitudes négatives de l'enseignant. Ces résultats concordent avec les écrits qui rapportent de très forts liens entre les difficultés d'apprentissage et les problèmes du comportement (Patterson, Forgatch, Yoerger et Stoolmiller, 1998). II semble, comme le rapportent Patterson, Reid et Dishion (1992), que les problèmes du comportement interfèrent avec l'apprentissage scolaire et qu'ils conduisent à l'échec scolaire. Il est fort probable qu'un élève qui vit des difficultés scolaires en arrive à se décourager puis à se désengager de ses activités scolaires. Enfin, leur faible niveau de coopération en classe se traduit par le non-respect des consignes et par une incapacité à se conformer aux demandes de l'enseignant. Ils ont de la difficulté à faire correctement leur travail scolaire et à remettre leurs travaux à temps. Puis l'enseignant développe des attitudes négatives envers l'élève, ce qui contribue à une mauvaise relation. Ensuite, l'élève peut percevoir les enseignants comme responsables de ses échecs tout en développant des attitudes négatives envers l'école. Enfin, la période qui précède le décrochage se traduit habituellement par un fort taux d'absentéisme. Dans ce contexte, il n'est pas surprenant que la faible performance scolaire et les problèmes du comportement sont des variables fortement associées au décrochage (Battin-Pearson et al., 2000). 
Les difficultés d'interaction sociale des élèves en problèmes du comportement ne sont probablement pas étrangères au fait que les enseignants les décrivent comme étant instables, agités, entêtés, désobéissants, immatures et non coopératifs. En fait, ces élèves, en plus d'être perturbateurs en classe, ne sont pas engagés dans leur réussite scolaire, ce qui cause de sérieux problèmes de gestion de classe. Il semble que la qualité de la relation enseignant-jeune soit très associée à la réussite et à la persévérance scolaire du jeune (Janosz et al., 1998; Potvin et al., 1999; Smyth et Hattam, 2002).

Le rôle des parents dans le processus du décrochage scolaire de leur enfant est de plus en plus documenté. Les résultats concernant les variables familiales suggèrent que les parents des élèves témoins et de ceux présentant des problèmes du comportement non décrocheurs se différencient des parents d'élèves en problèmes de comportement décrocheurs quant à une pratique éducative fortement associée aux problèmes du comportement: l'encadrement parental. Cette pratique éducative se traduit par la supervision des sorties et de l'occupation du temps de l'enfant. Le parent veut savoir ce que fait son enfant lorsqu'il est à l'extérieur de la maison et il veut connaître ses amis. Cette pratique éducative est très importante, car elle favorise la socialisation du jeune (Frick, 1998). La mauvaise qualité de l'encadrement parental des jeunes présentant des problèmes de comportement est rapportée dans de nombreuses études, ce qui porte à croire qu'il est peut être un facteur spécifique associé aux problèmes du comportement (Loeber, 1997; Patterson et al., 1992).

Une limite de notre étude est d'avoir évalué les perceptions des élèves et des enseignants uniquement à partir de mesures auto-rapportées. Des entrevues auprès des parents permettraient probablement de mieux comprendre les variables familiales qui différencient chaque type. Un protocole multi-évaluation et multi-site, comprenant des questionnaires, des entrevues et de l'observation, fournirait des connaissances plus complémentaires.

En conclusion, les problèmes de comportement représentent un facteur fortement associé au décrochage scolaire pour un sous-groupe d'élèves qui se caractérise par un cumul de facteurs de risque associés aux plans personnel, scolaire et familial. L'ensemble de ces facteurs suggère que ces élèves démontrent peu d'intérêt pour l'école tout en ne la valorisant pas. Ces résultats ont une implication pour les intervenants en milieu scolaire, car si un nombre important d'élèves qui présentent des problèmes de comportement décroche, un plus grand nombre ne décroche pas. Il est possible que ces derniers ne requièrent pas d'intervention spécifique. II est intéressant d'observer que les élèves en problèmes du comportement non décrocheurs obtiennent de bons résultats scolaires, sont assez coopératifs en classe et vivent un bon encadrement parental. De nouvelles études 
devraient permettre de bien identifier les sous-groupes de problèmes du comportement qui ont besoin d'intervention préventive de ceux qui n'en ont pas besoin.

\section{Références}

Battin-Pearson, S., Newcomb, M.D., Abbott, R.D., Hill, K.G., Catalano, R.F. et Hawkins, J.D. (2000). Predictors of early high school dropout: A test of five theories. Journal of Educational Psychology, 92(3), 568-582.

Beck, A.T. (1978). Depression inventory. Philadelphia: Center for Cognitive Therapy.

Bouchard, G., Sabourin, S., Lussier, Y., Richer, C. et Wright, J. (1995). Nature des stratégies d'adaptation au sein des relations conjugales: présentation d'une version abrégée du Ways of Coping Questionnaire. Revue canadienne des sciences du comportement, 27(3), 371-377.

Bourque, F. et Beaudette, D. (1982). Étude psychométrique du questionnaire de la dépression de Beck auprès d'un échantillon d'étudiants universitaires francophones. Revue canadienne des sciences du comportement, 14(3), 211-218.

Deslandes, R., Bertrand, R., Royer, É. et Turcotte, D. (1995). Validation d'instruments de mesure du style parental et de la participation parentale dans le suivi scolaire. Revue Mesure et évaluation en éducation, 18(2), 63-80.

Epstein, J.L., Connors, L.J. et Salinas, K.C. (1993). High school and family partnerships: Questionnaires for teachers, parents and students. Baltimore Johns Hopkins University, Center on School, Family and Community Partnerships.

Fortin, L. et Picard, Y. (1999). Les élèves à risque de décrochage scolaire: facteurs discriminants entre décrocheurs et persévérants. Revue des sciences de l'éducation, 25(2), 359-374.

Fortin, L., Royer, É., Marcotte, D., Potvin, P. et Joly, J. (2001). Épreuves de validité d'une mesure d'habiletés sociales auprès d'adolescents québécois à l'école secondaire. Psychologie et psychométrie, 22(1), 23-44.

Fortin, L., Royer, É., Potvin, P., Marcotte, D. et Yergeau, É. (2004). La prédiction du risque de décrochage scolaire au secondaire: facteurs personnels, familiaux et scolaires. Revue canadienne des sciences du comportement, 36(3), 219-231.

French, D.C. et Conrad, J. (2001). School dropout as predicted by peer rejection and antisocial behavior. Journal of Research on Adolescence, 11(3), 225-244.

Frick, P. J. (1998). Conduct disorders and severe antisocial behavior. New York: Plenum Press.

Garnier, H.E., Stein, J.A. et Jacobs, J.K. (1997). The process of dropping out of high school: A 19 years perspective. American Educational Research Journal, 34(2), 395-419.

Gouvernement du Québec (2000). Une école adaptée à tous ses élèves: Projet de politique de l'adaptation scolaire. Québec: Ministère de l'Éducation.

Gouvernement du Québec (2002). Indicateurs économiques. Québec: Ministère de l'Éducation.

Gouvernement du Québec (2004). Indicateurs de l'éducation. Québec: Ministère de l'Éducation, Bureau de la direction des statistiques et des études quantitatives.

Gresham, F.M. et Elliott, S.N. (1990). Social skills rating system. Circle Pines: American Guidance Service.

Janosz, M., Georges, P. et Parent, S. (1998). L'environnement éducatif à l'école secondaire: un modèle théorique pour guider l'évaluation du milieu. Revue canadienne de psychoéducation, 
27(2), 285-306.

Jimerson, S., Egeland, B., Sroufe, L.A. et Carlson, B. (2000). A prospective longitudinal study of high school dropouts examining multiple predictors across development. Journal of School Psychology, 38(6), 525-549.

Lazarus, R.S. et Folkman, S. (1984). Stress, appraisal and coping. New York: Springer.

LeBlanc, M. (1994). Questionnaire de la délinquance auto-révélée. Rapport de recherche. Montréal: Université de Montréal, Département de psychoéducation.

Lee, V.E. et Burkam, D.T. (2003). Dropping out of high school: The role of school organisation and structure. American Educational Research Journal, 40(2), 353-393.

Loeber, R. et Hay, D. (1997). Key issues in the development of aggression and violence from childhood to early adulthood. Annual Review of Psychology, 48, 371-410.

Lessard, A., Fortin, L., Joly, J., Royer, É., Potvin, P. et Marcotte, D. (2006). Les raisons de l'abandon scolaire: différences de genre. Revue québécoise de psychologie, 27(1), 135-152. (À paraitre au moment de la publication originale)

McNeal, Jr., R.B. (1999). Parental involvement as social capital: Differential effectiveness on science achievement, truancy and dropping out. Social Force, 78(1), 117-144.

Moos, R.H. et Trickett, E.J. (1987). Classroom Environment Scale Manual (2 éd.). Palo Alto: Consulting Psychologists Press.

Patterson, G.R., Forgatch, M.S., Yoerger, K.L. et Stoolmiller, M. (1998). Variables that initiate and maintain an early-onset trajectory for juvenile offending. Development and Psychopathology, 10(3), 531-547.

Patterson, G.R. Reid, J.B. et Dishion, T.J. (1992). Antisocial Boys. A social learning approach: IV. Eugene: Castalia Publishing Compagny.

Potvin, P., Deslandes, R., Beaulieu, P., Marcotte, D., Fortin, L., Royer, É. et Leclerc, D. (1999). Risque d'abandon scolaire, style parental et participation parentale au suivi scolaire. Revue canadienne de l'éducation, 24(4), 441-453.

Potvin, P. et Rousseau, R. (1991). Les attitudes réciproques des enseignants et des élèves en difficultés scolaires. Rapport de recherche subventionné par le Fonds pour la formation de chercheurs et l'aide à la recherche (FCAR EQ 3562). Trois-Rivières: Université du Québec à Trois-Rivières, Département de psychologie.

Smyth, J. et Hattam, R. (2002). Early school leaving and the cultural geography of high schools. British Educational Research Journal, 28(3), 375-397.

Steinberg, L., Lamborn, S.D., Dornbusch, S.M. et Darling, N. (1992). Impact of parenting practices on adolescent achievement: Authoritative parenting, school involvement and encouragement to succeed. Child Development, 63(5), 1266-1281.

Tobin, T.J. et Sugai, G.M. (1999). Using sixth-grade school records to predict school violence, chronic discipline problems and high school outcomes. Journal of Emotional and Behavioral Disorders, 7(1), 40-53.

Walker, H.M., Colvin, G. et Ramsey, E. (1995). Antisocial behavior in school: Strategies and best practices. Belmont: Pacific Grove/Cole Publishing Company. 